\title{
A New EEMD-Effective Transfer Entropy-Based Methodology for Exchange Rate Market Information Transmission in Southern Africa Development Community
}

\author{
Anokye M. Adam (D), ${ }^{1,2}$ Emmanuel N. Gyamfi ${ }^{D},{ }^{3}$ Kwabena A. Kyei, ${ }^{2}$ Simiso Moyo, ${ }^{2}$ \\ and Ryan S. Gill ${ }^{4}$ \\ ${ }^{1}$ Department of Finance, School of Business, University of Cape Coast, Cape Coast, Ghana \\ ${ }^{2}$ Department of Mathematics and Applied Mathematics, University of Venda, Thohoyandou, South Africa \\ ${ }^{3}$ GIMPA Business School, Ghana Institute of Management and Public Administration, Accra, Ghana \\ ${ }^{4}$ Department of Mathematics, University of Louisville, Louisville, KY, USA
}

Correspondence should be addressed to Emmanuel N. Gyamfi; engyamfi@gimpa.edu.gh

Received 30 September 2021; Accepted 1 November 2021; Published 16 November 2021

Academic Editor: Paulo Jorge Silveira Ferreira

Copyright (c) 2021 Anokye M. Adam et al. This is an open access article distributed under the Creative Commons Attribution License, which permits unrestricted use, distribution, and reproduction in any medium, provided the original work is properly cited.

\begin{abstract}
The desire to form monetary unions among regional blocs in Africa has necessitated the need to assess the degree of financial systems interdependencies in Africa economic blocs for their suitability to have harmonised economic policies of eventual monetary unions. In this regard, SADC has pursued policies to harmonise and integrate its financial system as a precursor to its intended monetary union. However, the ensuing risk among exchange rates of economies in SADC is presumed to rise during severe uncertainties. This study examines the degree of asymmetry and nonlinear directional causality between exchange rates in SADC in the frequency domain. We employ both the ensemble empirical mode decomposition (EEMD) and the Rényi effective transfer entropy techniques to investigate the multiscale information that might be disregarded and further quantify the directional flow of information. Analysis of the study is presented for four frequency-domains: high-, medium-, and low frequencies, representing short-, medium-, and long-terms, respectively, in addition to the residue (fundamental feature). We find a mixture of asymmetric and nonlinear bidirectional and unidirectional causality between exchange rates in SADC for the sampled period. The study reveals a significant positive information flow in the high frequency, but negative flow in the medium and low frequencies. In addition, we gauge a bidirectional significant negative information flow within all the 15 economies for the residue. This suggests a higher risk of uncertainties in exchange rates of SADC. Our findings for low probability events at multiscales have implications for the direction of the future of the SADC monetary union. This calls for further sustained policy harmonisation in the region.
\end{abstract}

\section{Introduction}

The desire to integrate economies of Africa regional blocs as enshrined in Article 3 of the Africa Union Constitution has necessitated the need to ensure coordination of macroeconomic factors. The anticipated benefits of such economic integration are well-researched and documented [1].

The exchange rate market integration has been cited as a key indicator for stable economic integration because of its pass-through effect on other financial markets [2]. The rising uncertainty in the global economic development makes the comovement of exchange rates important for the formation of monetary unions. The SADC formed in 1999 for decades has pursued policies to harmonise and integrate the financial system with the intention to form a monetary union. This hinges on the perceived benefits of membership in a monetary union, thus, stronger exchange rate, low exchange risk, and price stability.

Several studies have attempted to depict and determine precisely the linear and nonlinear linkages between 
exchange rate markets aiming to form a monetary union as the understanding of the interconnectivity is important for the conduct of monetary policy and how to deal with activities of market participants. Among related studies, the linkage of the exchange rate in West Africa Monetary Zones [3-5] has been severally investigated. Within the SADC bloc, several studies have attempted to model the interaction of exchange rate markets [1, 2, 6-9]. Except for Adam et al. [1] and Adam et al. [2], the identified studies seldom studied the comovement of the exchange rate from a multiscale perspective. The exchange rate market is a complex system with varying participants with varying objectives, investment preferences, and motives as depicted by the heterogeneous market hypothesis $[1,2,9-11]$. Thus, exchange rate data could be noisy, nonstationary, nonlinear, and mixed $[12,13]$. These intrinsic complexities of exchange rate data question the appropriateness of the use of static models in exchange rate studies.

Accordingly, recent studies on coordination of exchange rate markets have relied on models that can extract better high frequency signals about the exchange rate to deal with its apparent noisy behaviour to provide better understanding $[1,2,5,14-16]$. This is aimed at analysing exchange rate markets from the time-frequency domain perspective instead of the traditional time-domain viewpoint. The Fourier and wavelet transform approaches of studying the time-frequency domain have been widely used in this regard [17-19]. Huang et al. [20] noted that Fourier-based approaches are not data-adaptive, unable to capture the timevarying characteristics of the neural signal, and only designed for the frequency analysis of stationary time series. The wavelet transform is however counterintuitive in its interpretation and also nonadaptive.

The empirical mode decomposition (EMD) by Huang et al. [20] provides a new perspective of analysing nonlinear and nonstationary data in the time-frequency domain based on the direct extraction of signal energy associated with various intrinsic time scales. The EMD is adaptive, fully posterior, and physically meaningful which makes it superior to its alternatives [20,21]. In addition, because the EMD process is completely based on the local time scales of time series, with no prior basis, the extracted oscillations reflect the time series accurately. However, the conventional EMD suffers from the problem of mode-mixing which is corrected by the ensemble empirical mode decomposition (EEMD) proposed by $\mathrm{Wu}$ and Huang [22].

The extant literature on exchange rate markets comovements has relied on spillover index, cross-correlation, the Granger causality test, the vector autoregressive model, and the generalised autoregressive conditional heteroskedasticity model. The many limitations associated with the applications of these econometric models emanating from the need to satisfy stationarity and distributional properties hamper their applications. The transfer entropy proposed by Schreiber [23] comes in handy to quantify information transfer or information flow between variables in a system with no assumptions about the distribution or intercorrelation of the original variables. The transfer entropy is effective in identifying linear and nonlinear relationships between variables, is asymmetric and built upon transition probability, is practical in all systems, and does not require prior specification model $[24,25]$.

In this paper, we propose EEMD-Effective Transfer Entropy- (EEMD-ETE-) based methodology to analyse exchange rate markets information transmission. The effective transfer entropy is based on Rényi transfer entropy [26]. The EEMD-ETE methodology provides two novelties in studying exchange rates comovement compared to previous studies. First, it examines exchange rate markets from the perspective of information transmission and quantification to capture information spillover and interactions among different markets, which provides useful information on the spillover direction between variables [27]. Second, financial time series often exhibit different characteristics at different time frequencies, and the relations between different variables vary widely across time scales $[28,29]$. The utilization of EEMD-ETE, therefore, offers the opportunity to understand the extent of information transmission at different frequency scales. Last, it delineates the influence of noise from the quantification of information flow across the exchange rate markets.

We find a mixture of asymmetric and nonlinear bidirectional and unidirectional causality between exchange rates in SADC for the sampled period. The study reveals a significant negative information flow in the medium (medium-term) and low frequencies (long-term), but a more positive flow in the high frequency (short-term). However, from the fundamental feature represented by the residue, we gauge a bidirectional significant negative information flow within all the 15 economies. This suggests a higher risk of uncertainties in exchange rates of SADC.

The rest of the paper is structured as follows. Section 2 introduces the methods employed in the study; Section 3 describes the exchange rate data of SADC used in the study and Section 4 presents the results and analysis. Section 5 highlights the policy implications and conclusion.

\section{Methodology}

We initially present the EEMD technique, followed by the transfer entropy. Thus, the outcome generated from the EEMD is used as input data for the effective transfer entropy estimations.

\subsection{Ensemble Empirical Mode Decomposition. The EEMD is} an improvement of the EMD-based signal processing method to solve the easy mode-mixing effect of EMD. The EMD is a dyadic filter bank in the frequency domain [30]. The goal of the empirical mode decomposition is to decompose the original data (nonstationary and nonlinear data) into IMFs and a residue. The EMD is a fully datadriven decomposition method and IMFs are derived directly from the signal itself. As indicated by Huang et al. [20], an IMF must satisfy two criteria:

(i) The number of extrema and the number of zero crossings must either be equal or differ at most by one. 
(ii) At any point, the mean value of the envelope is defined by the local maxima and the envelope defined by the local minima is zero.

The first condition forces an IMF to be a narrow-band signal with no riding waves. The second condition ensures that the instantaneous frequency will not have fluctuations arising from an asymmetric waveform [20].

The IMFs are obtained through a process called the sifting process which uses local extrema to separate oscillations starting with the highest frequency. Given a time series $x(t), t=1,2,3, \ldots, M$, the process decomposes it into a finite number of functions, denoted as $\operatorname{IMF}_{k}(t), k=1,2,3, \ldots, n$ and a residue $r_{n}(t)$. The residue is the nonoscillating drift of the data. If the decomposed data consist of uniform scales in the frequency space, the EMD acts as a dyadic filter and the total number of IMFs is approximately equal to $n=\log _{2}(N)$ [30]. At the end of the decomposition process, the original time series can be reconstructed as

$$
x(t)=\sum_{i=1}^{m} \operatorname{IMF}_{k}(t)+r_{m}(t) .
$$

The EEMD makes the signal be of continuity at different scales by the uniform distribution feature of the Gaussian white noise frequency. The noises are offset by multiple averaging processing to inhibit and even eliminate noise influence [31, 32]. For a time series $x(t)$, the EEMD includes the following steps:

(i) Generate a new signal of $y(t)$ by superposing to $x(t)$ a randomly generated white noise with an amplitude equal to a certain ratio of the standard deviation of $x(t)$.

(ii) Perform the EMD algorithm on $y(t)$ to obtain the IMFs

(iii) Repeat steps 1 to 2 for $m$ times with different white noise to obtain an ensemble of IMFs

$$
\begin{aligned}
& \left\{\operatorname{IMF}_{k}^{1}(t), k=1,2, \ldots, n\right\}, \\
& \left\{\operatorname{IMF}_{k}^{2}(t), k=1,2, \ldots, n\right\}, \ldots,\left\{\operatorname{IMF}_{k}^{m}(t), k=1,2, \ldots, n\right\} .
\end{aligned}
$$
(iv) Calculate the average of IMFs
$\left\{\mathrm{IMF}_{k}(t), k=1,2, \ldots, n\right\}$, where

$$
\left\{\overline{\operatorname{IMF}_{k}(t)}=\frac{1}{m \sum_{i}^{m} \operatorname{IMF}_{k}^{i}(t)}\right\} .
$$

The importance of the process is that the observed data are a combination of true time series and noise and that the ensemble means of data with different noises are closer to the true time series. Therefore, the addition of white noise as an additional step to the steps in the EMD process may help to extract the true IMF by offsetting the noise through ensemble averaging [33].

\subsection{Measuring Information Flows Using Rényi Transfer} Entropy. Before we discuss the Rényi transfer entropy, we present the concept of Shannon entropy as a measure of uncertainty upon which transfer entropy is embedded in information theory $[34,35]$. We consider a probability distribution with diverse results of a given experiment $p_{j}$. Following Hartley [36], each symbol's average information is specified as

$$
H=\sum_{j=1}^{n} P_{j} \log _{2}\left(\frac{1}{P_{j}}\right) \text { bits, }
$$

where $n$ signifies number of distinct symbols concerning the probabilities $P_{j}$.

The concept of entropy, later referred to as Shannon entropy, was introduced in 1948 by Shannon [37]. It proffers that, for a discrete random variable $(J)$ with probability distribution $(P(j))$, the average number of bits needed to optimally encode independent draws [34] can be presented as

$$
H_{J}=-\sum_{j=1}^{n} P(j) \log _{2} P(j) .
$$

Shannon entropy is connected with the concept of Kullback-Leibler distance [38] to assess the information flow between two time series with the concept of Markov processes. We present $I$ and $J$ as two discrete random variables with corresponding marginal probabilities of $P(i)$ and $P(j)$, with joint probability $P(i, j)$, with dynamic structures in line with a stationary Markov process of order $k$ (Process $I$ ) and $I$ (process $J$ ). The Markov property implies that the probability to observe $I$ at time $t+1$ in state $i$ conditional on the $k$ prior observations is $p\left(i_{t+1} \mid i_{t}, \ldots, i_{t-k+1}\right)=p\left(i_{t+1} \mid i_{t}, \ldots, i_{t-k}\right)$. To encode the observation in $t+1$, the average bits number required once the ex-ante $k$ values are known can be illustrated as

$$
h_{j}(k)=-\sum_{i} P\left(i_{t+1}, i_{t}^{(k)}\right) \log P\left(i_{t+1} \mid i_{t}^{(k)}\right),
$$

where $i_{t}^{(k)}=\left(i_{t}, \ldots, i_{t-k+1}\right)$ (correspondingly for process $J$ ). In a bivariate perspective as well as relying on the KullbackLeibler distance [38], information transmission from process $J$ to process $I$ is measured by quantifying the deviation from the generalised Markov property $P\left(i_{t+1} \mid i_{t}^{(k)}\right)=P\left(i_{t+1} \mid i_{t}^{(k)}, j_{t}^{(I)}\right)$. The Shannon transfer entropy can thus be presented as

$$
T_{J \longrightarrow I}(k, l)=\sum P\left(i_{t+1}, i_{t}^{(k)}, j_{t}^{(I)}\right) \log \frac{P\left(i_{t+1} \mid, i_{t}^{(k)}, j_{t}^{(I)}\right)}{P\left(i_{t+1} \mid, i_{t}^{(k)}\right)},
$$

where $T_{J \longrightarrow I}$ calculates the information flow from $J$ to $I$. Analogously, $T_{I \longrightarrow I}$, as a measure for the information flow from $I$ to $J$, can be derived. Calculating the differential can reveal the prevalent direction of information flow between $T_{J \longrightarrow I}$ and $T_{I \longrightarrow J}$.

Based on the Shannon entropy so far discussed, we present the Rényi transfer entropy [26] which is contingent on a weighting factor $q$ and can be calculated as

$$
H_{J}^{q}=\frac{1}{1-q} \log \sum_{j} P^{q}(j),
$$


with $q>0$. For $q \longrightarrow 1$, Rényi entropy converges to Shannon entropy. For $0<q<1$, thus, low probability events receive more weight, while for $q>1$ the weights benefit outcomes $j$ with a higher original probability. As a result, Rényi entropy permits accentuating diverse distribution areas, depending on factor $q[34,35]$.
Applying the escort distribution [39] $\varnothing_{q}(j)=p^{q}(j) / \sum_{j} p^{q}(j)$ with $q>0$ to normalize the weighted distributions, Rényi transfer entropy [26] is derived as

$$
\mathrm{RT}_{J \longrightarrow I}(k, l)=\frac{1}{1-q} P\left(i_{t+1}, i_{t}^{(k)}, j_{t}^{(I)}\right) \log \frac{\sum_{i} \varnothing_{q}\left(i_{t}^{(k)}\right) P^{q}\left(i_{t+1} \mid i_{t}^{(k)}\right)}{\sum_{i, j} \varnothing_{q}\left(i_{t}^{(k)}, j_{t}^{(I)}\right) P^{q}\left(i_{t+1} \mid i_{t}^{(k)}, j_{t}^{(I)}\right)}
$$

It is worth noting that the Rényi transfer entropy calculation can have negative results. Knowing the history of $J$, in this case, indicates considerably more uncertainty than knowing the history of $I$ alone would indicate.

The transfer entropy estimates are biased in small samples [40]. The correction of the bias is possible and can be used to calculate the effective transfer entropy as

$$
\operatorname{ETE}_{J \longrightarrow I}(k, l)=T_{J \longrightarrow I}(k, l)-T_{J \text { shuffled } \longrightarrow I}(k, l),
$$

where $T_{\text {Jshuffled } \longrightarrow I}(k, l)$ depicts the transfer entropy using a shuffled version of the time series $J$ that is randomly drawing values from the observed time series $J$ and realigning them to generate a new time series, which destroys the time series dependencies of $J$, not forgetting the statistical dependencies between $J$ and $I$. This enjoins $T_{J \text { shuffled } \longrightarrow I}(k, l)$ to come together to zero with increasing sample size, and any nonzero value of $T_{\text {Jshuffled } \longrightarrow I}(k, l)$ is due to small sample effects. To produce a bias-corrected effective transfer entropy estimate, repeated shuffling, and the average of the shuffled transfer entropy estimates overall replications serve as an estimator for the small sample bias, which is removed from the Shannon or Rényi transfer entropy estimate.

Relying on a Markov block bootstrap, the statistical significance of the transfer entropy estimates, as given by equation (10), can be assessed [41]. This preserves the dependencies within the variables $J$ and $I$ but eliminates the statistical dependencies between them contrary to shuffling. Repeated estimation of transfer entropy then provides the distribution of the estimates under the null hypothesis of no information flow. The associated $p$-value is given by $1-\widehat{q}_{T}$, where $\widehat{q}_{T}$ denotes the quantile of the simulated distribution that is determined by the respective transfer entropy estimate [35].

\section{Data Description}

We utilized daily real exchange rates of 15 out of 16 member countries of the SADC from 3rd January 1994 to 7 th January 2019, obtained from Thomson Reuters Datastream in this study. Specifically, daily data for local currency per USD for Angola, Botswana, Comoros, Democratic Republic of Congo, Eswatini (formerly Swaziland), Lesotho, Madagascar, Malawi, Mauritius, Mozambique, Namibia, Seychelles, South Africa, Tanzania, and Zambia were employed. The study period and countries were informed by data availability and the period South Africa formed part of SADC. As a result of these criteria, Zimbabwe was expunged from the analysis. The real exchange rate was measured based on nominal domestic currency per US dollar (USD) multiplied by the domestic consumer price index divided by the US consumer price index. The justification for using USD as a proxy currency is due to the dominance of the USD in international trade by these countries and the extent of dollarization of most SADC economies. In spite of recent dedollarization in Angola, Mozambique, and Zambia, the dollar remains dominant in international trade globally including SADC and provides a means of standardizing units of pair of currencies [42]. The study was executed with $R$ packages libeemd [43] for EEMD and RTransferEntropy for ETE $[35,44]$.

From Figure 1, the graphical presentation of the logarithm of exchange rates of SADC economies is shown, with the exception of Zimbabwe. Almost all the exchange rates trend upwards except Angola. The trajectory behaviour is explained by periods of pegging of the Angola Kwanza to the US dollar. The upward trend of remaining other countries shows that SADC exchange rates have over the period depreciated against the US dollar. It is not quite surprising to experience this upward trend because, generally, SADC countries are overvalued, and as expected, equilibrium is established overtime. Again, there is a biased expectation of the future exchange rates in small samples in times of uncertainty about when a future policy change will be implemented [45]. This partially explains the 'peso problem' proposition for the trend movements.

\section{Results and Analysis}

The analysis of this study was structured to follow three processes. First, we decomposed the daily real domestic currency/USD exchange rate to obtain individual 11 IMFs for each country's exchange rate and trend using EEMD. We examined the properties of all IMFs extracted and were found to satisfy the necessary and sufficient conditions to be IMFs as prescribed by Huang et al. [20]. The residue is the nonoscillating drift of the data, which is not affected by short- to medium-term fluctuations but by the structural changes in the data generation process. It, thus, represents the long-term trend of the data and for this study long trend behaviour of the exchange rate dictated by fundamentals of the economies. Second, the mean and amplitude of the IMFs 

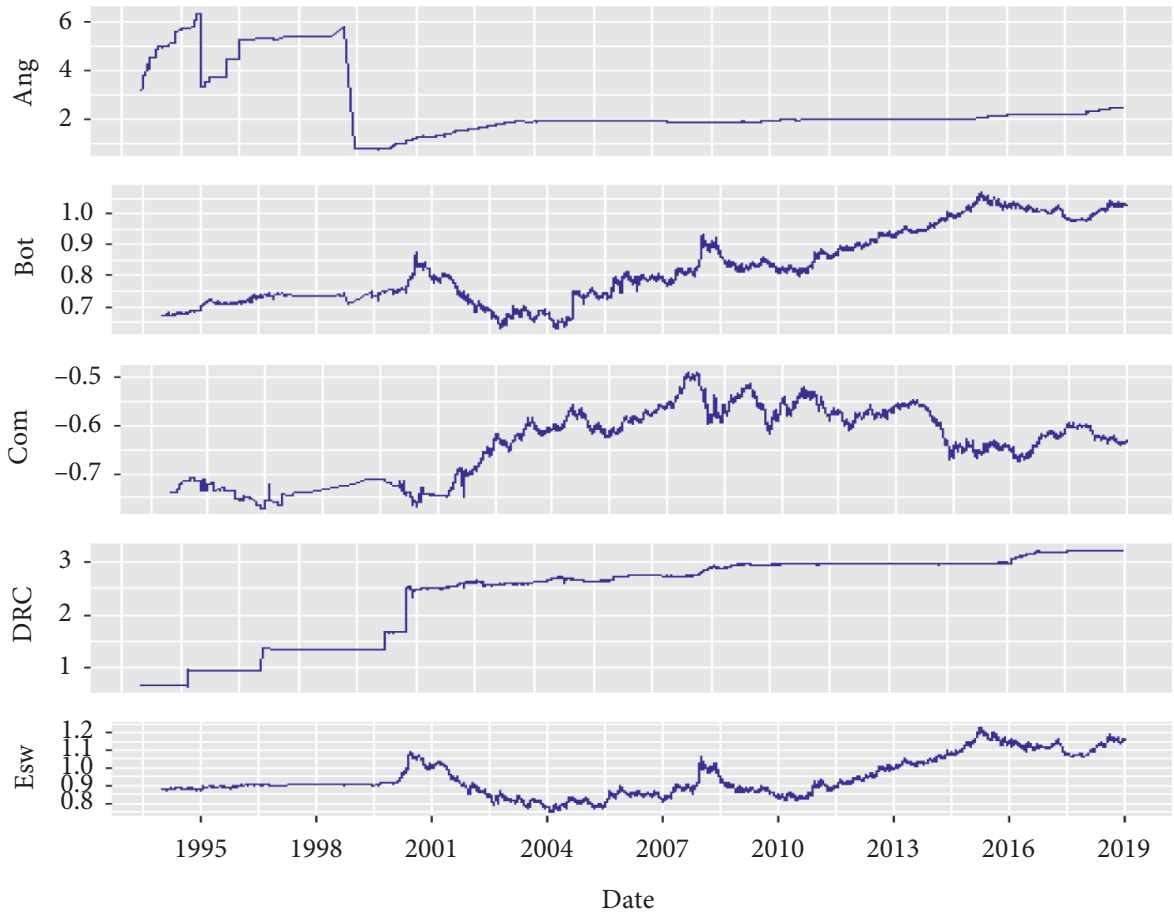

(a)
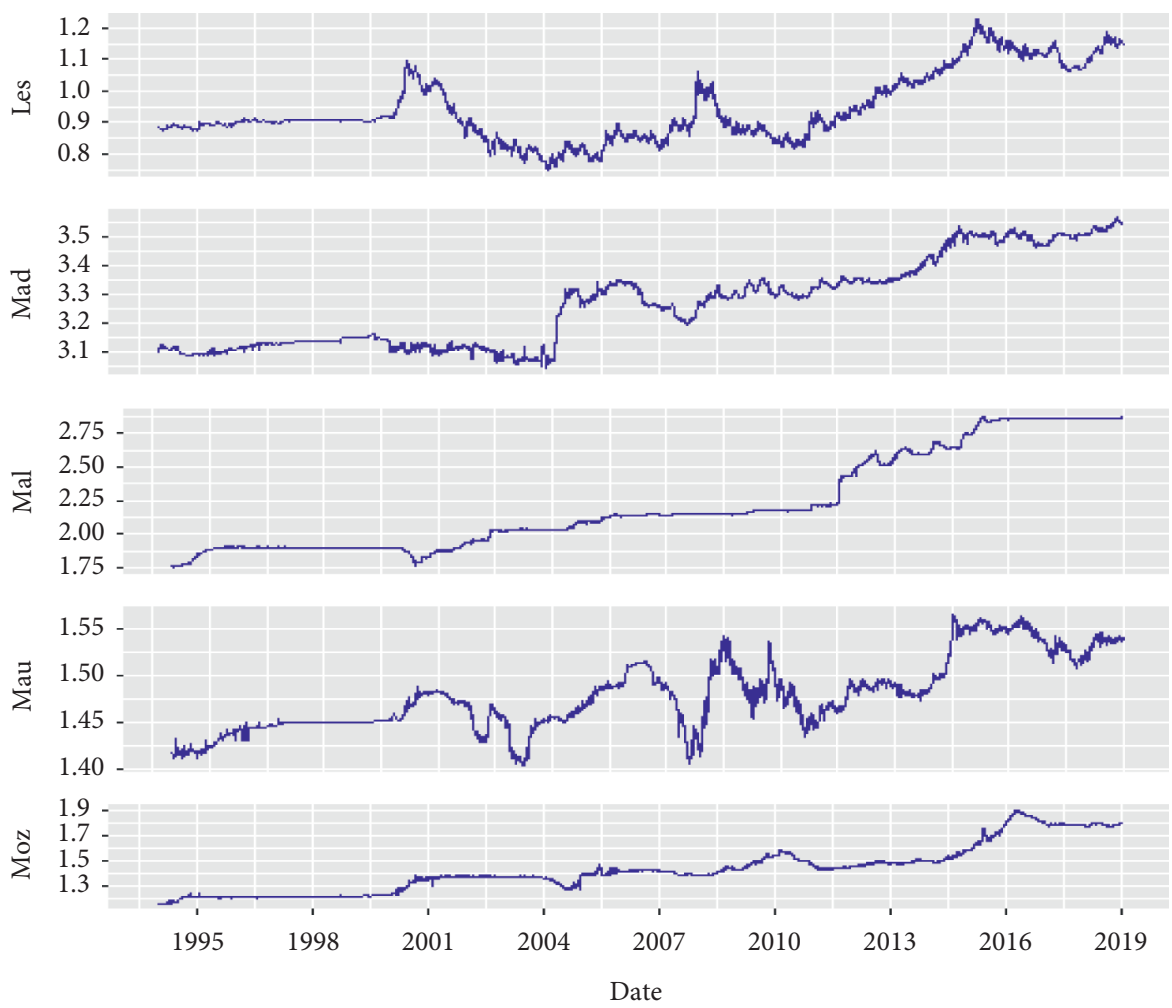

(b)

Figure 1: Continued. 

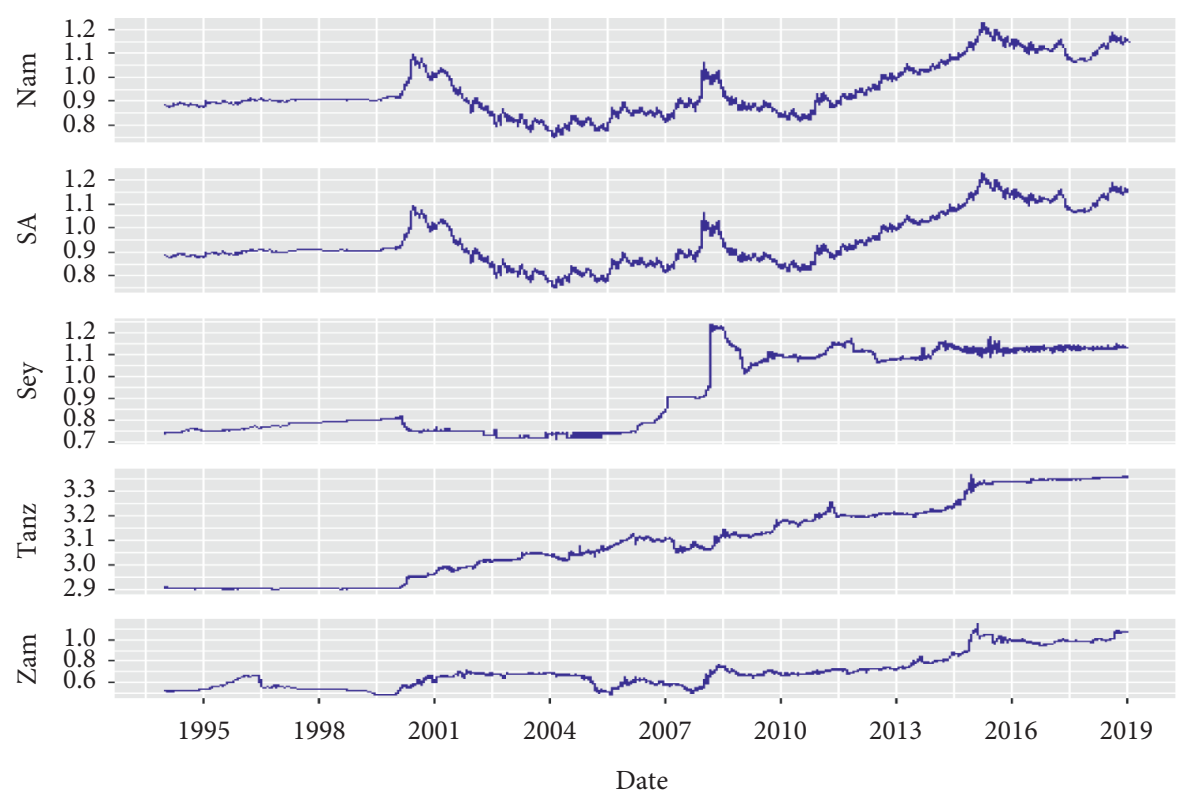

(c)

Figure 1: Plots of log of the daily real exchange rate of 15 countries of SADC (Figure 1 is reproduced from Adam et al. [1]).

of each country were classified into various frequencies: high frequency (sum of IMFs 1-5), medium frequency (sum of IMFs 6-8), and low frequency (sum of IMFs 9-11) using cluster analysis. The high, medium, and low frequencies have mean time-frequency of less than 30 , between a month and 12 months and more than 12 months, respectively.

Table 1 presents the clusters of IMFs into high (period of 1-15 days), medium (up to 144 days), and low frequencies (up to 2374 days) in addition to the residue. The Pearson product-moment and Kendall tau-b correlations between each frequency and the original data series, the variance percentage of each frequency in the original data series, and the sum of all frequencies and residue indicate that the residue is the dominant mode in all cases.

Last, to understand the flow of information at various frequencies for all countries related to the observed exchange rates, we further examine the Rényi effective transfer entropy of the logarithm of daily real exchange rate series, high-, medium-, and low frequencies, and residue components of 15 exchange rate market in SADC at $q=0.3$ to account for low probability events.

We present the bidirectional EEMD-ETE estimates in addition to the $95 \%$ confidence bounds between exchange rates in SADC at various frequencies. The frequencies indicate the importance of multiscales in financial time series. Thus, the dynamics of exchange rates do not occur immediately, but at several investment horizons as provided by the heterogeneous market hypothesis $[2,10]$.

The presence of a negative ETE implies that awareness of the exchange rate from a particular country suggests a higher risk coverage for the exchange rate in another country. A positive ETE indicates that the knowledge of the exchange rates reduces the risk of the exchange rate of a specific country. The knowledge in the tails is assigned a high weight for low values of $q$, resulting in a significant effective transfer entropy result in the current situation. For this reason, we set $q$ from the Rényi effective transfer entropy to 0.3 to offer more weights to the tail events, which bears direct implications. The ETE decreases and even becomes negative as the weight is reduced.

The Rényi effective transfer entropy emphasises various sections of the involved probability density functions in a nonlinear way. The Rényian transfer entropy is specifically used in this study to account for tail events associated with the dynamics of exchange rates movements within SADC. Since transfer entropy is a nonparametric estimate and has a higher likelihood of determining statistical interdependence between time series, we present the discussion between exchange rates of SADC following the concept of interdependencies. Doyle [46] postulated that when there is interdependence, it results in increased economic relations among countries which promotes the forming of unions. Furthermore, Polachek [47] argues that countries gain from interdependence, for example, the diverse advantages obtained from trading with other nations which most governments try to sustain. This may not be far from SADC which has pursued policies to harmonise and integrate the financial system with the intention to form monetary union. The purpose of the analysis is to ascertain whether SADC could form a reliable monetary union with stronger exchange rate and low exchange rate risk and ensure price stability.

Analyses of the study are presented for fifteen SADC economies concerning exchange rate which is important for trade and investments. The decomposed returns series are presented for the high, medium, and low frequencies, representing short-, medium-, and long-terms, respectively. In addition, we present the residue which denotes the 


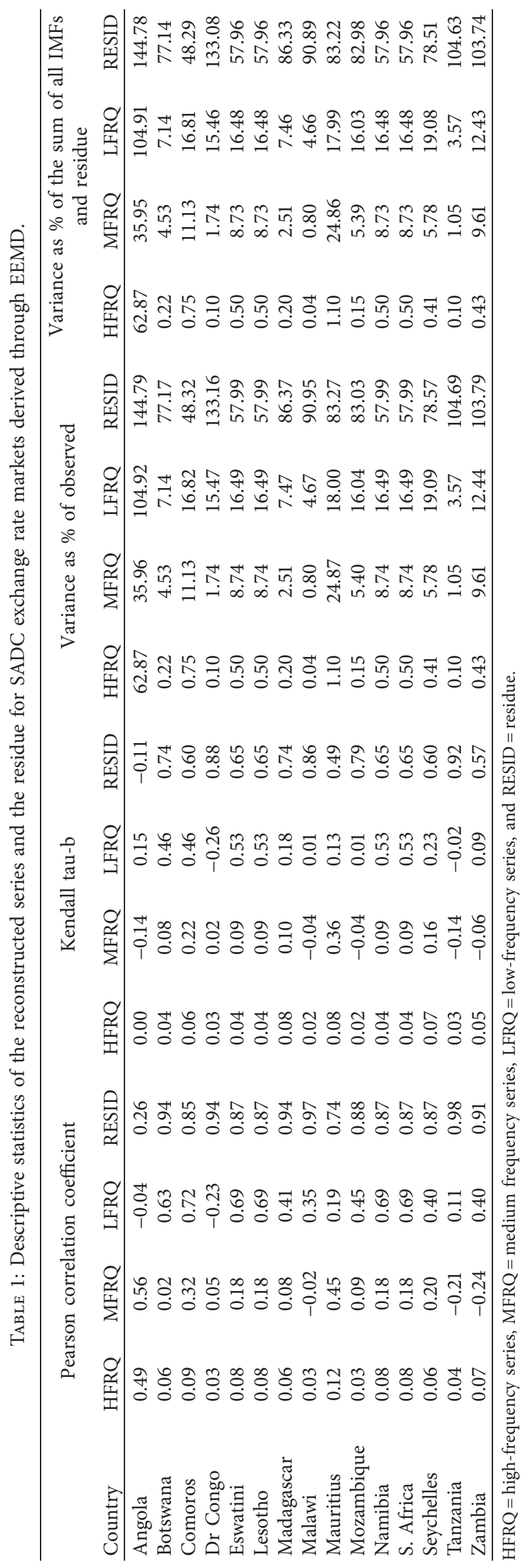


nonoscillating drift of the data, which is not affected by short- to medium-term fluctuations but by the structural changes in the data generation process. It, therefore, represents the trend behaviour of the exchange rate dictated by fundamentals of the economies. The final outputs are tail dependent and reveal the directional flow of information between exchange rates other than the ones shown by other statistical techniques which assumes linearity and stationarity. Consequently, Mokoena et al. [48] make it clear that SADC economies' exchange rates exhibit nonlinear relationships when purchasing power parity was assessed.

\subsection{Exchange Rates Information Transfer at High Frequency.} Figure 2 shows the information flow between exchange rates of SADC for high frequencies representing short-term horizon. It can be analysed that significant information flows between exchange rates within SADC are either positive or negative. However, there are more positive flows as compared to negative flows in the high frequency. Thus, the knowledge of the exchange rates from countries reduces the risk of the exchange rate of a specific country in the shortterm. This is also true for information flow from a specific country's exchange rate to the remaining SADC exchange rates. These observations imply that quantification of information flow between exchange rates in SADC depicts less uncertainties in the short-term. In other words, the knowledge of the history of one country's exchange rate illustrates considerably less uncertainty than knowing the history of only the remaining exchange rate(s).

Specifically, we find a bicausality positive significant information flow with countries such as Angola, Botswana, Comoros, Democratic Republic of Congo, Madagascar, Malawi, and Mauritius. This suggests that trade and investment within Angola, Botswana, Comoros, Democratic Republic of Congo, Madagascar, Malawi, and Mauritius with the remaining SADC economies would reduce exchange rate risk. Consequently, the presence of these economies in SADC with the quest of forming a reliable monetary union for stronger exchange rate and low exchange rate risk and ensuring price stability can suffice in the short-term. In this regard, economies of like nature may form a reliable monetary union with less shocks from a specific country's exchange rate. The findings for the short-term perspective corroborate the outcome of Zehirun et al. [8] who found a weak positive comovement in exchange rates in SADC when Johansen's multivariate cointegration technique was employed.

\subsection{Exchange Rates Information Transfer at Medium} Frequency. Figure 3 shows the information flow between exchange rates at SADC for medium frequencies representing a medium-term horizon. Overall, there is a more negative significant flow of information in the mediumterm. Thus, the knowledge of the exchange rates from countries increases the risk of the exchange rate of a specific country. This is also true for information flow from a specific country's exchange rate to the remaining SADC exchange rates. These observations imply that quantification of information flow between exchange rates in SADC indicates more uncertainties in the medium-term. In other words, the knowledge of the history of one country's exchange rate illustrates considerably more uncertainty than knowing the history of only the remaining exchange rate(s) as compared to the high frequency estimates.

Specifically, negative significant information flows from the exchange rates of Botswana, Eswatini, Namibia, South Africa, Democratic Republic of Congo, and Lesotho to Angola. The reverse is true except for information flow from Angola to the Democratic Republic of Congo. That is, there is bidirectional causality between exchange rates of Botswana, Eswatini, Namibia, South Africa, Lesotho, and Angola. Also, significant negative information flows from Lesotho, Eswatini, Namibia, South Africa, Zambia, Seychelles, Angola, and Mauritius to Botswana, with the reverse indicating a similar outcome which exhibits bidirectional causality. This suggests that trade and investment within Lesotho, Eswatini, Namibia, South Africa, Zambia, Seychelles, Angola, and Mauritius by Botswana would pose a higher risk for the exchange rate of Botswana, and vice versa. Relatively, trade and investment by Angola indicate less negative information flow from other countries as compared to Botswana. Furthermore, trade and investment to and from Zambia within SADC exhibit a higher degree of uncertainty with countries such as Botswana, Lesotho, South Africa, Eswatini, and the Democratic Republic of Congo. The outcome for trade and investment to and from SA with countries such as Lesotho, Namibia, Eswatini, and Botswana is no exception. The exchange rate negative information flow from South Africa to other SADC economies concurs with the findings of Qabhobho et al. [49] when GARCH models were considered to assess exchange rate volatilities. Accordingly, the presence of these economies in SADC with the quest of forming a reliable monetary union may not augur well for stronger exchange rates, low exchange rate risk, and ensuring price stability in the medium-term. In this regard, knowing the history of one country's exchange rate demonstrates considerably more uncertainty than knowing the history of only the remaining economies exchange rates, especially with the bidirectional significant negative causality case.

On the other hand, countries with insignificant unidirectional or, preferably, bidirectional insignificant or positive significant information flows may experience stronger exchange rate and low exchange rate risk and may result in price stability. For instance, the exchange rate dynamics of Tanzania depicts less risk of information sharing with the remaining SADC members in this study. In this regard, economies of like nature may form a reliable monetary union with fewer shocks from a specific country's exchange rate in the medium-term.

4.3. Exchange Rates Information Transfer at Low Frequency. Figure 4 shows the information flow between exchange rates at SADC for low frequencies representing a long-term horizon. Throughout the low frequency, there are more potentials for negative flows than positive flows between the 

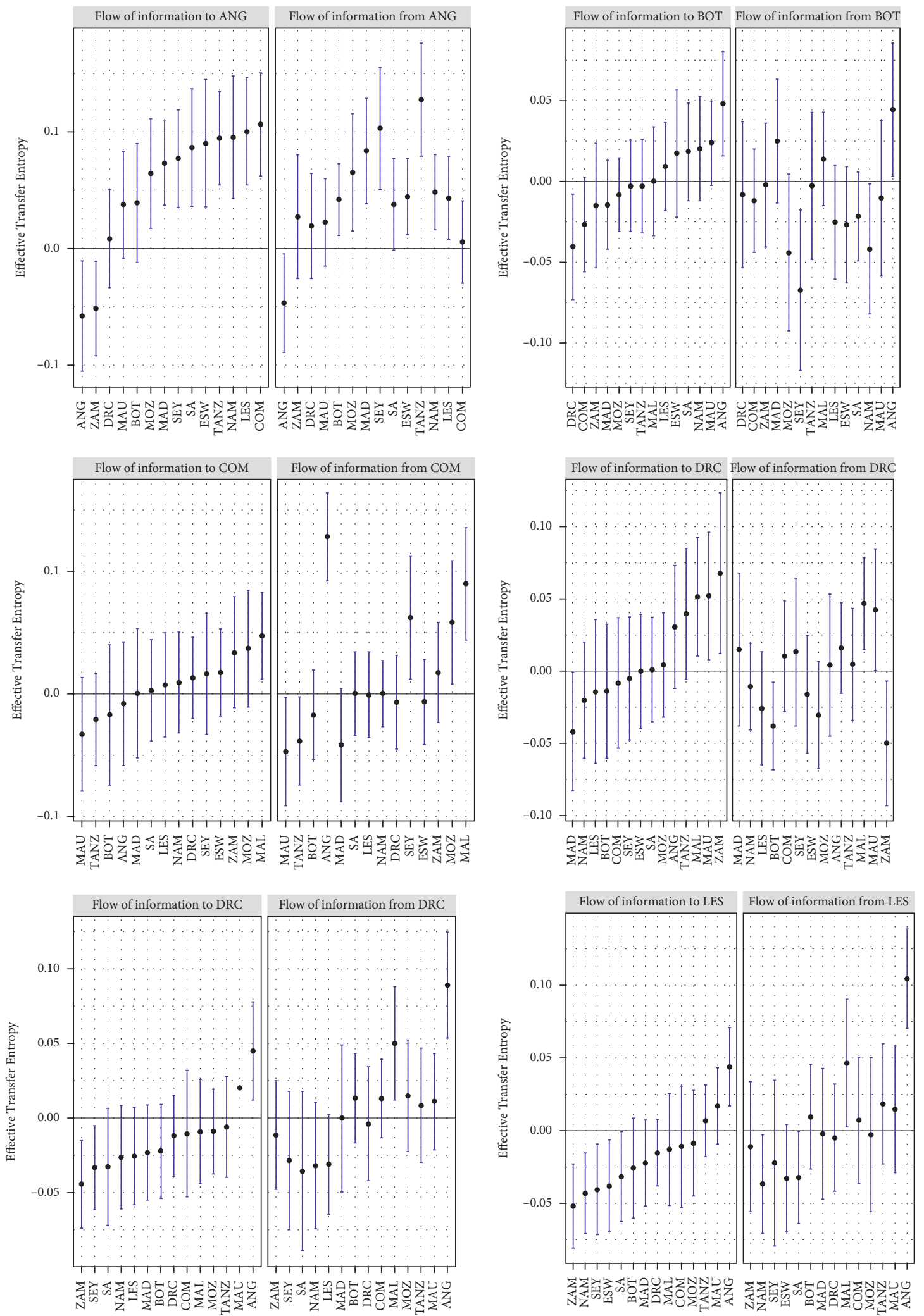

(a)

FIgURE 2: Continued. 

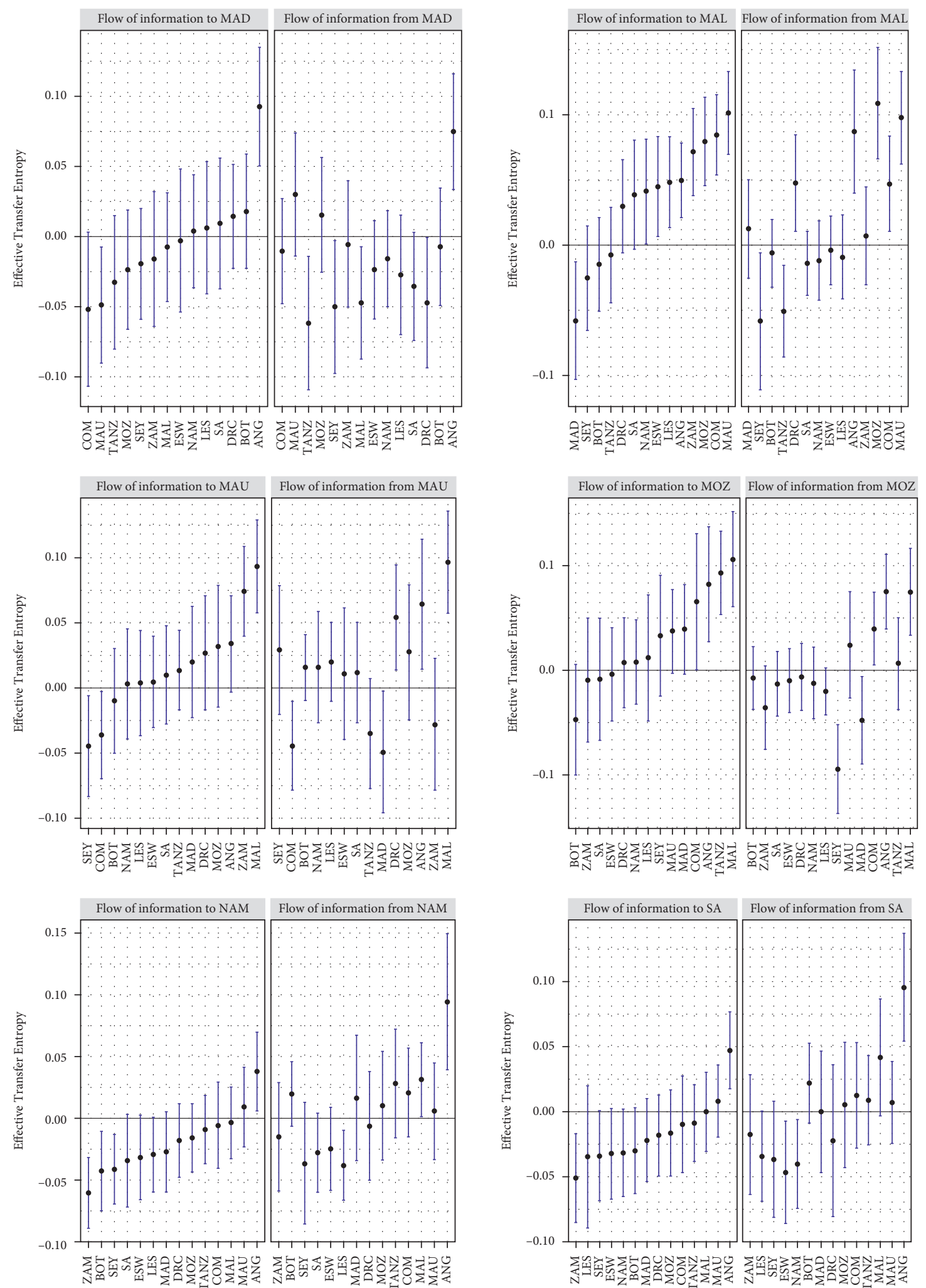

(b)

Figure 2: Continued. 

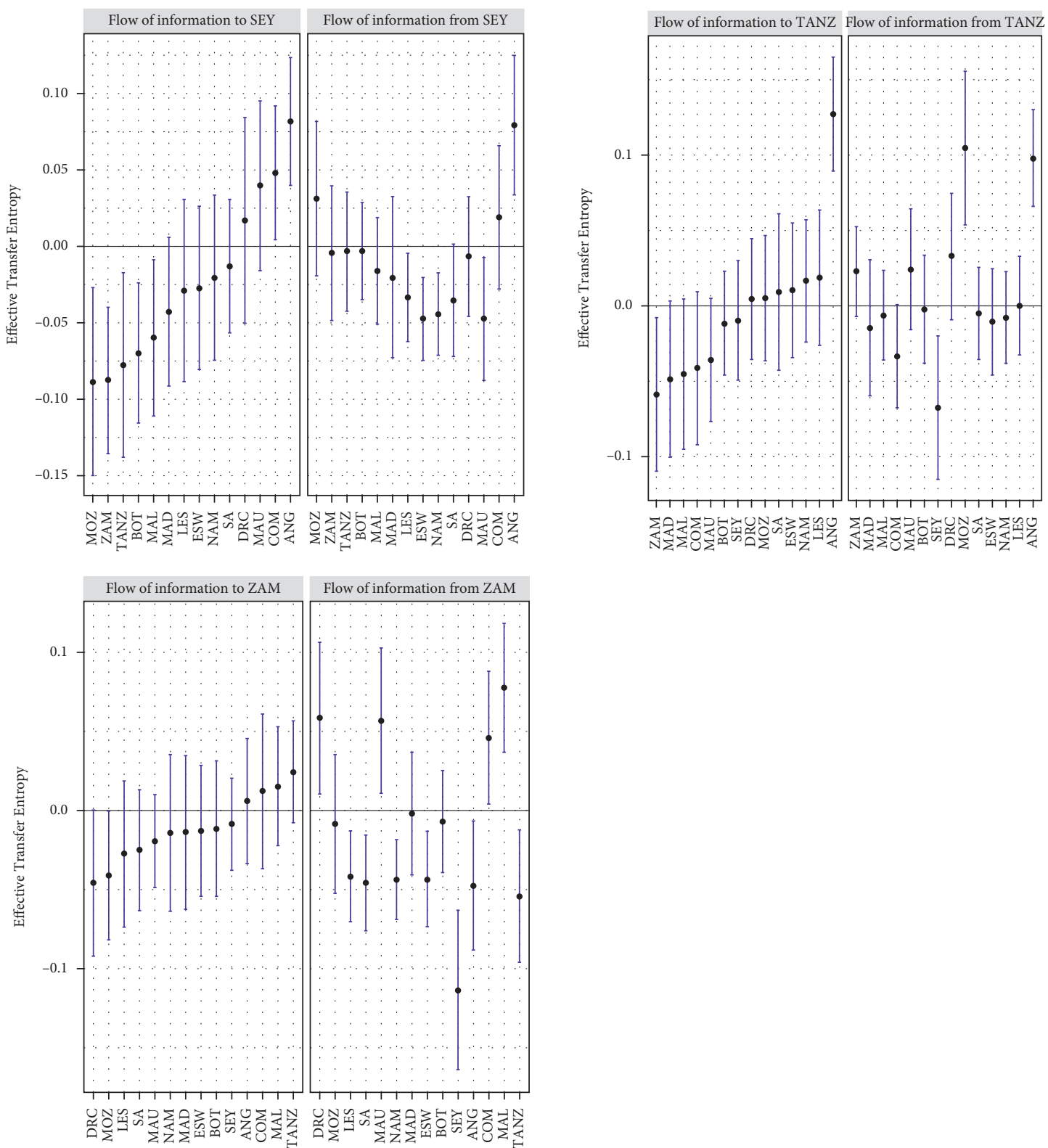

(c)

Figure 2: Information flow between exchange rates at high frequency series.

exchange rates in SADC. Thus, the knowledge of the exchange rates from countries increases the risk of the exchange rate of a specific country. This is also true for information flow from a specific country's exchange rate to the remaining SADC exchange rates. These observations imply that quantification of information flow between exchange rates in SADC depicts more uncertainty.

Specifically, negative significant information flows from the exchange rates of Democratic Republic of Congo to Angola; Eswatini to Botswana; South Africa, Lesotho, Namibia, and Mauritius to Eswatini; South Africa, Lesotho, Namibia, and Mauritius to Eswatini; Namibia, Eswatini, South Africa, and Mauritius to Lesotho; Angola to Mozambique; South Africa, Eswatini, Lesotho, and
Mauritius to Namibia; Lesotho, Namibia, Eswatini, and Mauritius to South Africa; Malawi and Tanzania to Seychelles; and South Africa, Lesotho, Eswatini, Malawi, Comoros, and Botswana to Zambia. We found a bidirectional causality with the exchange rates of South Africa, Lesotho, Namibia, Mauritius, and Eswatini. These economies exhibit negative significant information flow in exchange rates with respect to their possible combinations. Consequently, the inclusion of these economies as part of SADC with the quest of forming a reliable monetary union may not auger well for stronger exchange rate, low exchange rate risk, and ensuring price stability. The negative bidirectional causality further signifies that the knowledge of the exchange rates among these countries increases the risk of 

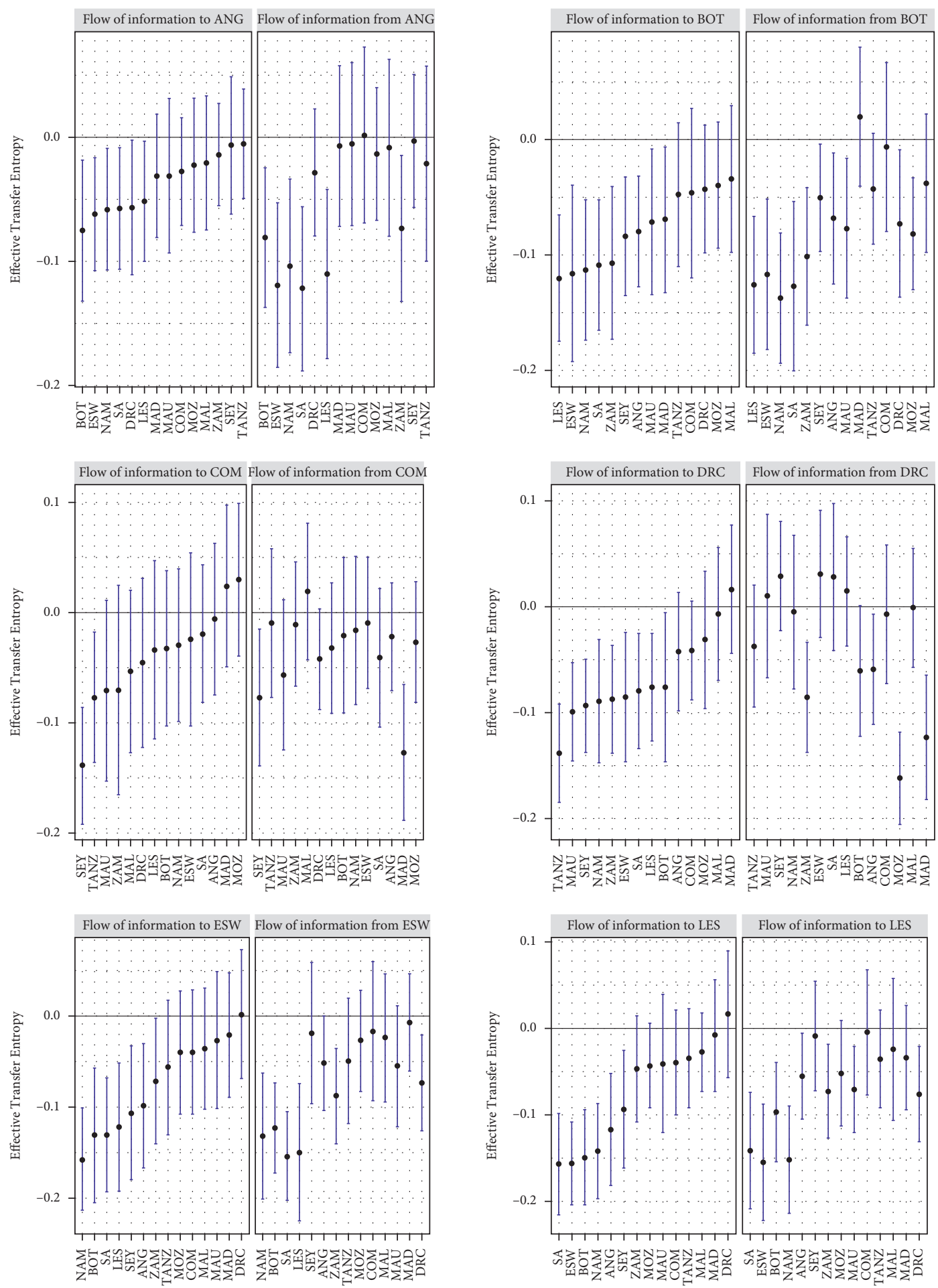

(a)

Figure 3: Continued. 

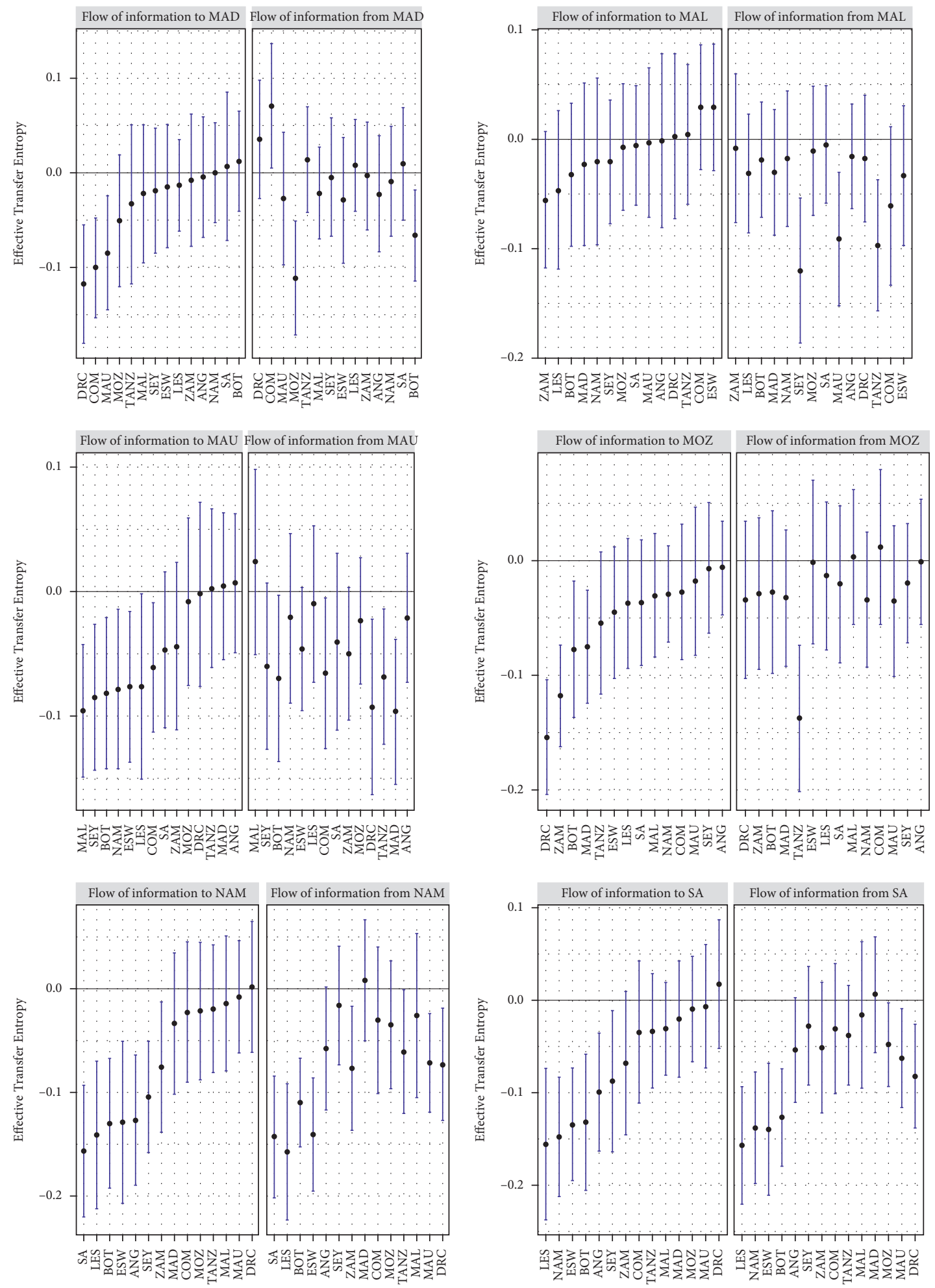

(b)

FIGURE 3: Continued. 

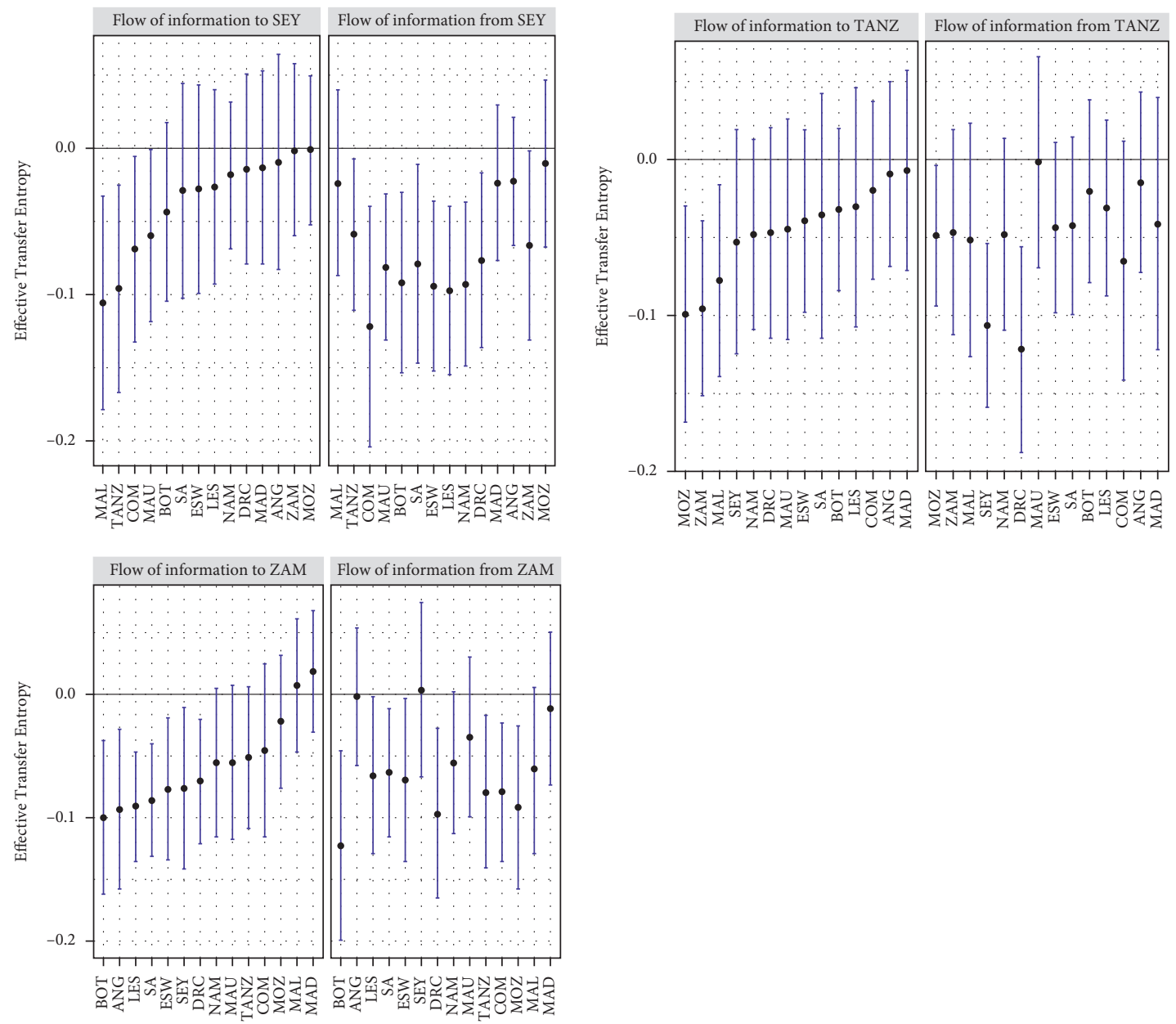

(c)

Figure 3: Information flow between exchange rates at medium frequency series.

the exchange rates within these regions. Thus, at low probability events, adverse fluctuations in exchange rates of SADC occur relative to favourable outcomes.

Accordingly, countries with a mixture of insignificant unidirectional/bidirectional flow or, preferably, bidirectional positive information flow may experience stronger exchange rate and low exchange rate risk, which may result in price stability. For instance, the exchange rates of Madagascar, Tanzania, and Malawi, to mention but a few, depict less uncertainties of information sharing. The outcome for Madagascar, Tanzania, and Malawi supports the assertion made by Anoruo and Ahmad [50] of monetary convergence in SADC. In this regard, economies of like nature may form a reliable monetary union with less shocks from a specific country's exchange rate.

4.4. Exchange Rates Information Transfer between the Residues. Figure 5 shows the information flow between exchange rates at SADC for the residue representing the long-term trend or fundamental feature. It can be observed from the plots that the residue contains the most negative significant information flow between exchange rates relative to the remaining frequencies. Thus, the knowledge of the exchange rates from countries increases the risk of the exchange rate of a specific country. This is also true for information flow from a specific country's exchange rate to the remaining SADC exchange rates. These observations imply that information flow between exchange rates in SADC indicates more uncertainties. Thus, the knowledge of the history of one country's exchange rate illustrates considerably more uncertainty than knowing the history of only the remaining exchange rate(s) as compared to the high-, medium-, and low frequencies estimates. This assertion confirms the suggestion of Duma [51] that SADC does not form an optimum currency union. The study of Redda and Muzindusti [52] advocated that bilateral real exchange rates in the SADC region share a common stochastic trend in the long-run, without considering the directional flow of information at multiscales between the economies. Thus, similarities in stochastic trends are not enough to prove, empirically, the conditions for optimum currency area. Notwithstanding, the study of Adam et al. [1, 2] found similarities in exchange rates of SADC at diverse frequencies via the EEMD approach. Building upon these similarities, our approach (EEMD-ETE) reveals a significant negative 

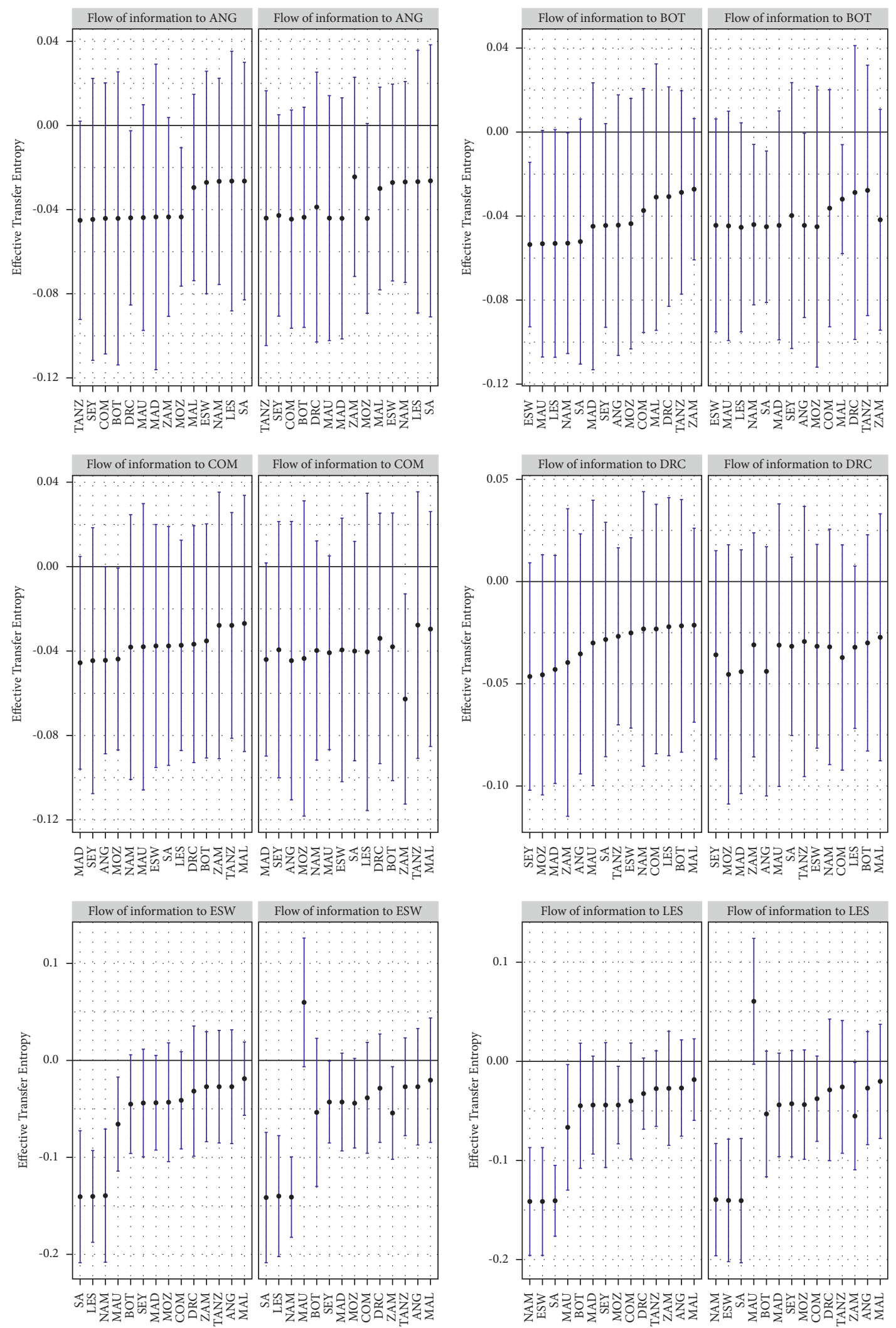

(a)

Figure 4: Continued. 

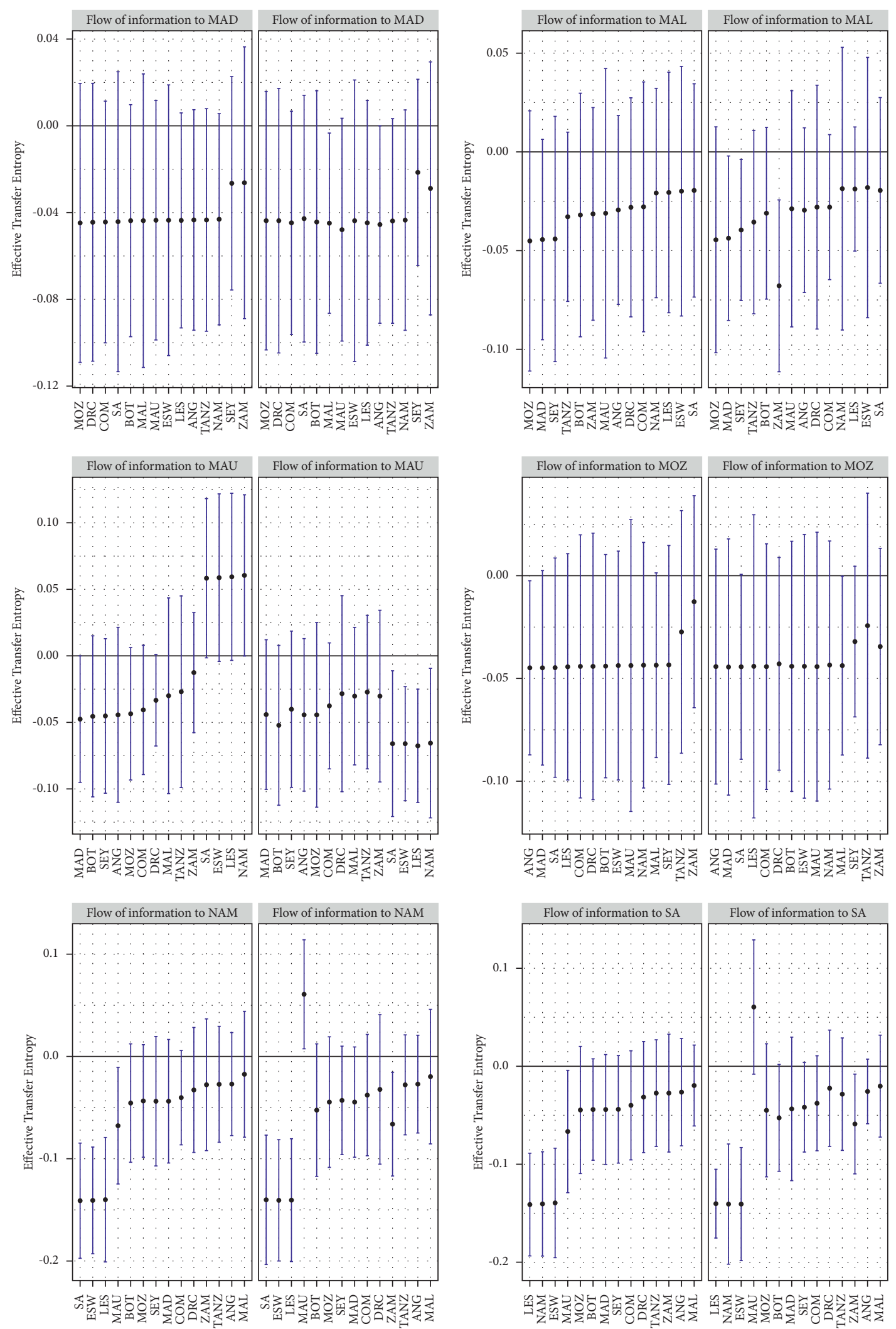

(b)

Figure 4: Continued. 

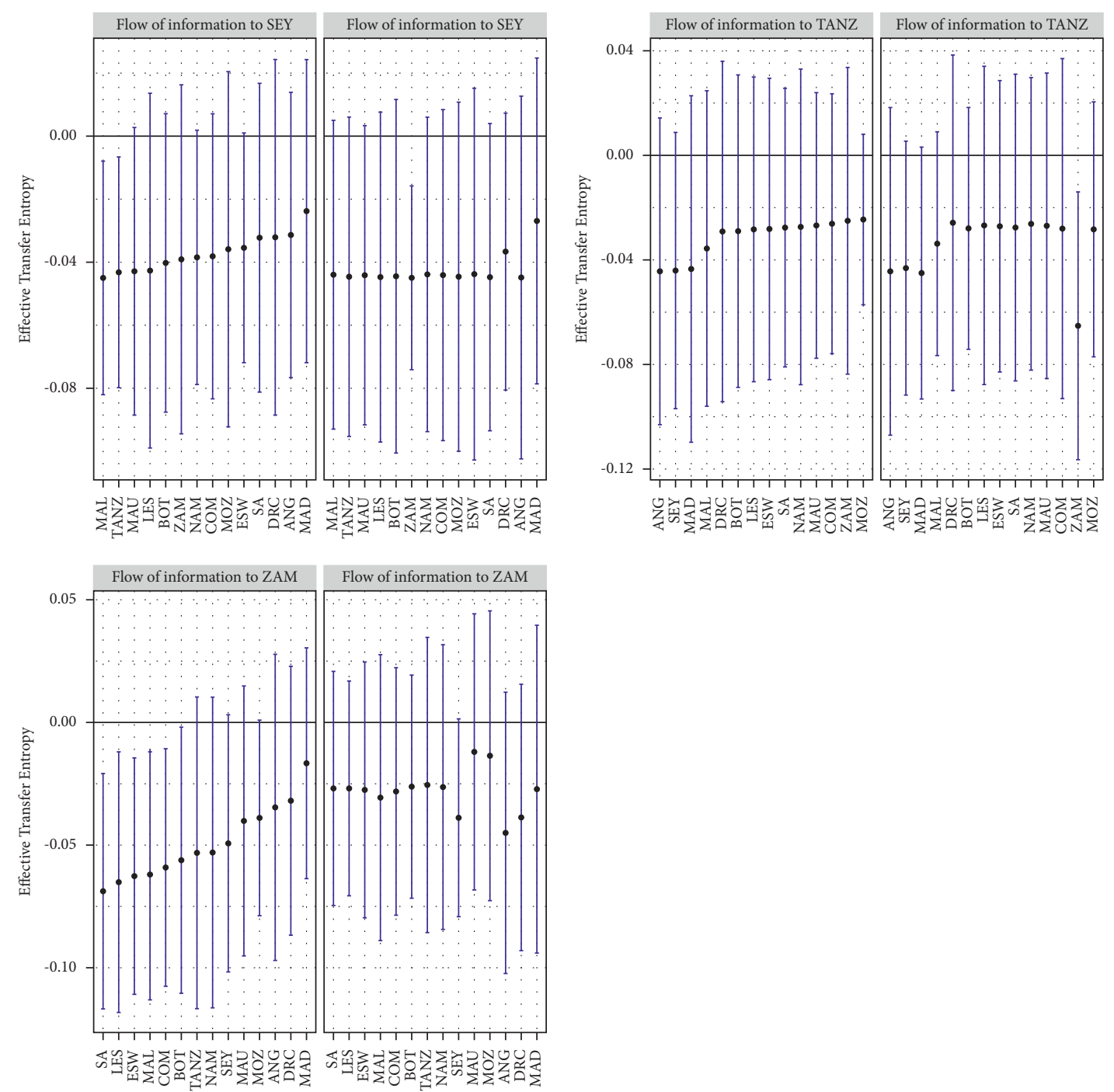

(c)

FIgURE 4: Information flow between exchange rates at low-frequency series.

information flow between the exchange rates of SADC for most scales.

A glance from the plots depicts that almost all the 15 SADC economies demonstrate negative information flow between exchange rates. During this period, trade and investments between union members may escalate exchange rate risk, weaken exchange rates, and lead to price instability.
In this regard, knowing the history of one country's exchange rate demonstrates considerably more uncertainty than knowing the history of only the remaining economies exchange rates, especially, from the bidirectional negative information flow. The significant negative bidirectional causality between exchange rates in SADC supports the findings of Zehirun et al. [8] in the long-run. 

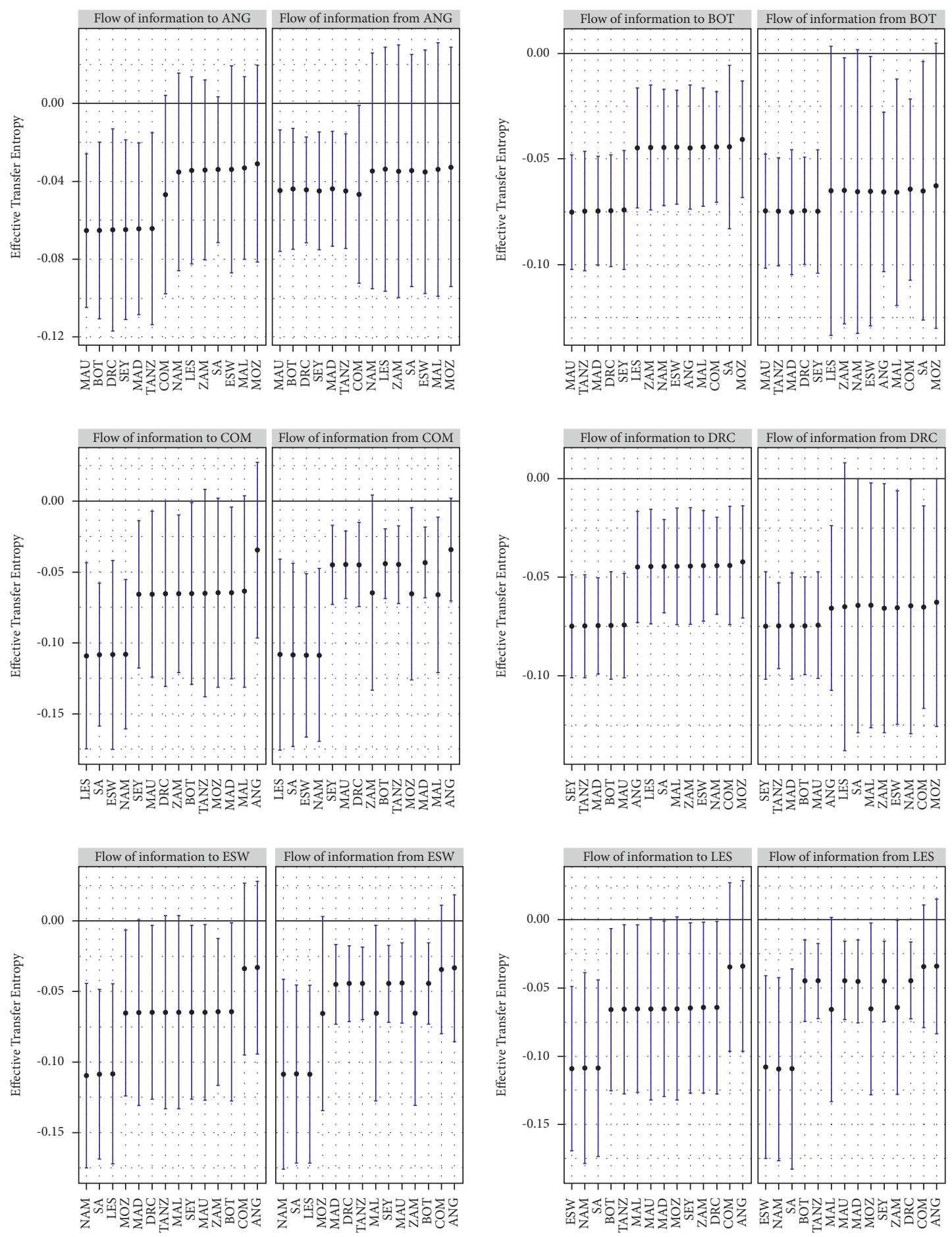

(a)

Figure 5: Continued. 

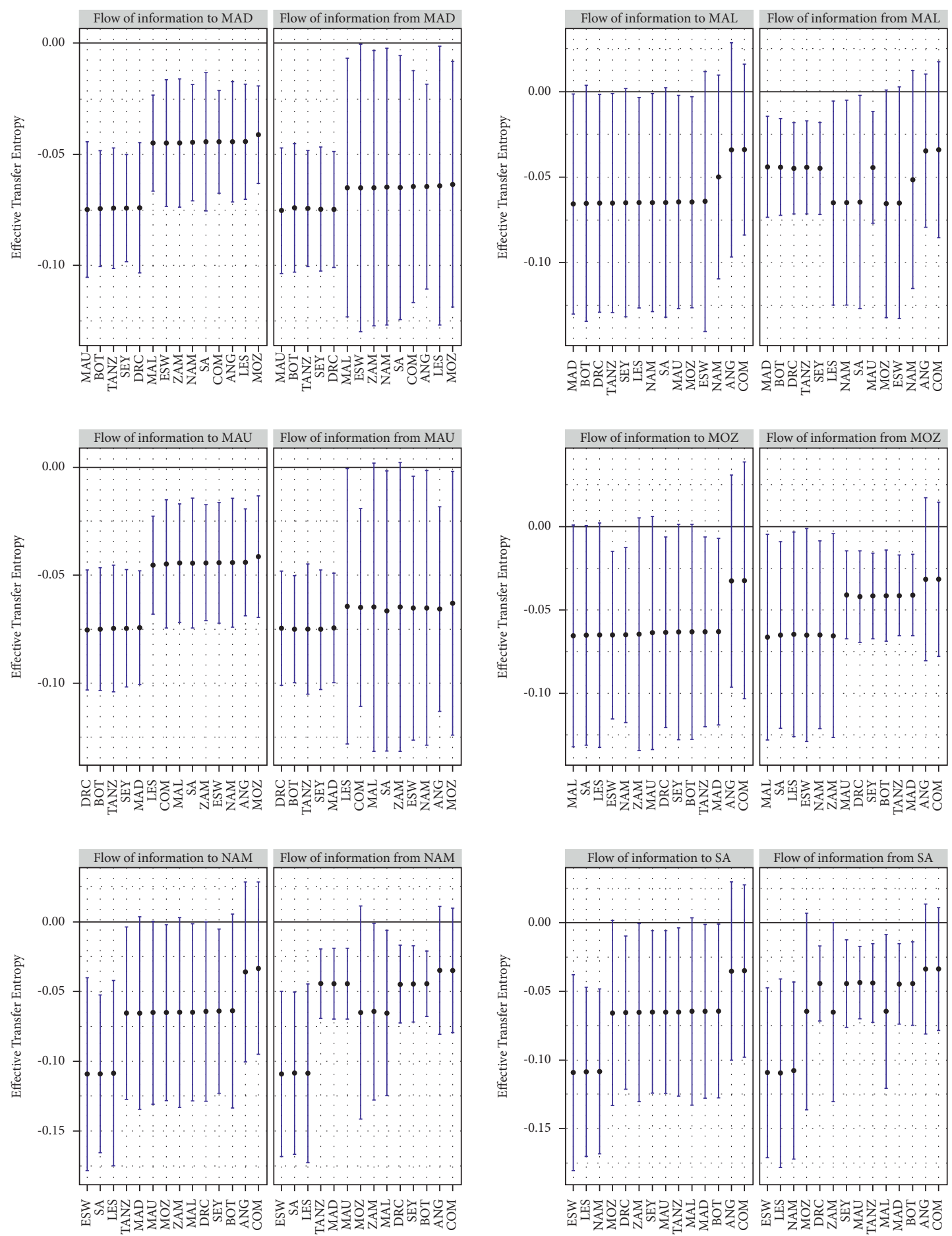

(b)

Figure 5: Continued. 

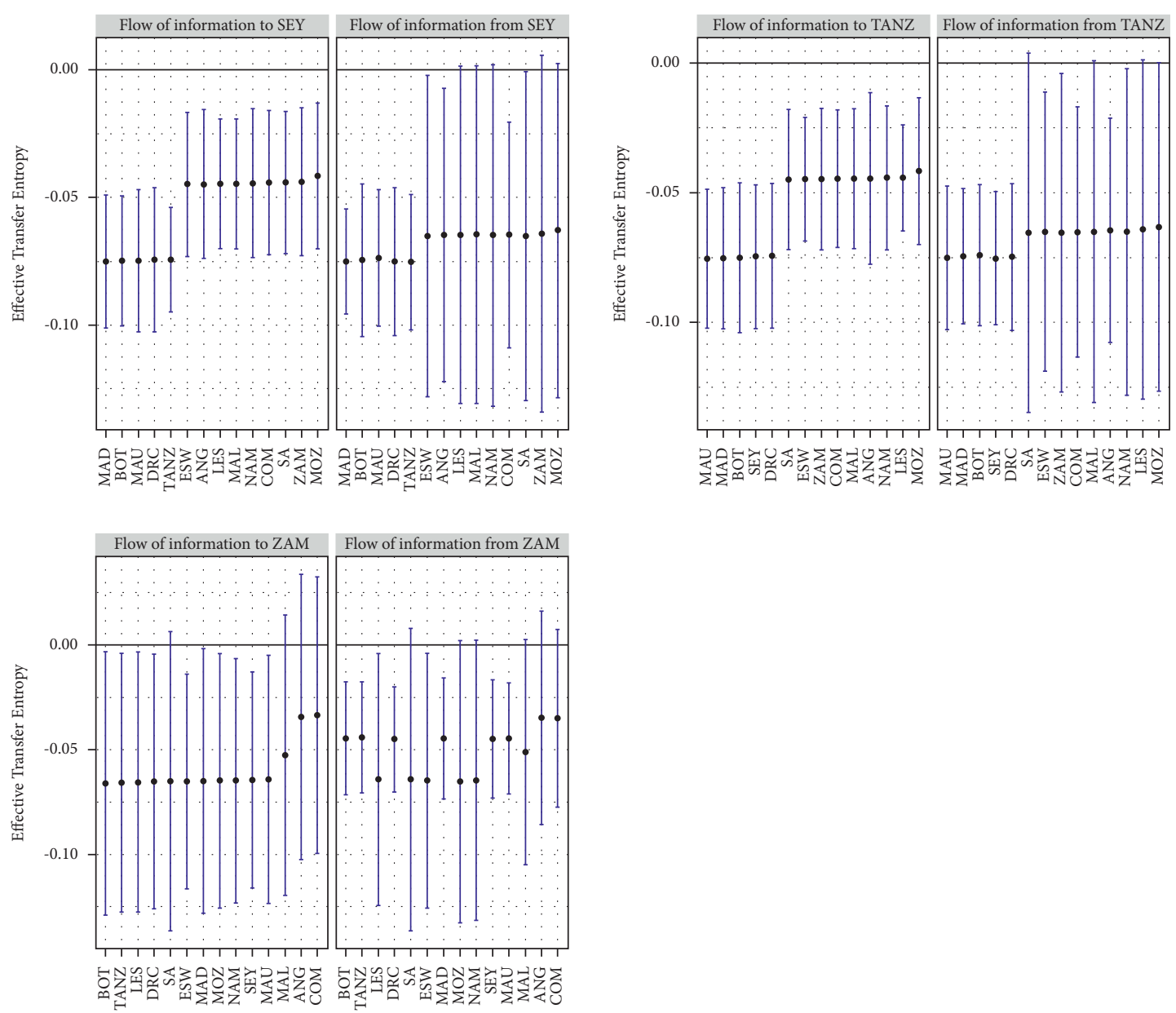

(c)

Figure 5: Information flow between exchange rates at the residue.

\section{Conclusion}

The EEMD-ETE was utilized in this study to quantify the direction and strength of information transfer between exchange rates at the frequency domain. In this regard, we investigated the multiscale information that might be disregarded. Owing to the nonlinearity of most financial time series, we adopt a log-likelihood ratio transfer entropy which quantifies information from a probability density function. We set $q$ from the Rényi transfer entropy to 0.3 to account for extreme events, specifically, low probability events. This indicates that it is tail events rather than observations in the centre that becomes imperative to be studied when information flow is employed.

Analysis of the study was presented for four frequencydomains, these are high-, medium-, and low frequencies, in addition to the trend. We find a mixture of asymmetric and nonlinear bidirectional and unidirectional causality between exchange rates in SADC for the sampled period. The study reveals a significant negative information flow in the medium- and long-terms, but a more positive flow in the shortterm (high frequency). However, at the residue (fundamental feature), we gauge a bidirectional significant negative information flow within all the 15 economies. This suggests a higher risk of uncertainties in exchange rates of SADC.

Our findings for low property events at multiscales have policy implications for the direction of the future of SADC monetary union. This would require tough decisions concerning monetary and exchange rate policies. It is not surprising to see the adverse information flow between exchange rates in SADC since most SADC economies have floating exchange rates and an independent monetary policy. To have a sound system of monetary union, a period of exchange rate convergence would be essential, with all potential SADC members pursuing an agreed exchange rate policy. This would gradually minimise the adverse exchange rate fluctuations between these economies in SADC over a given time.

\section{Data Availability}

The data are available publicly.

\section{Conflicts of Interest}

The authors declare that they have no conflicts of interest. 


\section{References}

[1] A. M. Adam, K. Kyei, S. Moyo, R. Gill, and E. N. Gyamfi, "Multifrequency network for SADC exchange rate markets using EEMD-based DCCA," Journal of Economics and Finance, 2021.

[2] A. M. Adam, K. Kyei, S. Moyo, R. Gill, and E. N. Gyamfi, "Similarities in southern african development community (SADC) exchange rate markets structure: evidence from the ensemble empirical mode decomposition," Journal of African Business, vol. 2021, Article ID 1874795, 16 pages, 2021.

[3] P. Alagidede, G. Tweneboah, and A. M. Adam, "Nominal exchange rates and price convergence in the West African monetary zone," International Journal of Business and Economics, vol. 7, no. 1, p. 181, 2008.

[4] R. Adu, I. Litsios, and M. Baimbridge, "Real exchange rate and asymmetric shocks in the west african monetary zone (WAMZ)," Journal of International Financial Markets, Institutions and Money, vol. 59, pp. 232-249, 2019.

[5] P. Owusu Junior, A. M. Adam, and G. Tweneboah, "Comovement of real exchange rates in the west african monetary zone," Cogent Economics \& Finance, vol. 5, no. 1, p. 1351807, 2017.

[6] T. D. Agbeyegbe, "On the feasibility of a monetary union in the southern Africa development community," International Journal of Finance \& Economics, vol. 13, no. 2, pp. 150-157, 2008.

[7] Y. Khamfula and H. Huizinga, "The southern african development community: suitable for a monetary union?" Journal of Development Economics, vol. 73, no. 2, pp. 699-714, 2004.

[8] M. F. Zehirun, M. C. Breitenbach, and F. Kemegue: Assessment of Monetary Union in SADC: Evidence from Cointegration and Panel Unit Root Tests.

[9] M. F. Zerihun, M. C. Breitenbach, and F. Kemegue, "Exploring exchange rate based policy coordination in SADC," Studies in Economics and Finance, vol. 33, 2016.

[10] U. A. Müller, M. M. Dacorogna, R. D. Davé, O. V. Pictet, R. B. Olsen, and J. R. Ward, Fractals and Intrinsic Time: a Challenge to Econometricians, Unpublished Manuscript, Olsen \& Associates, Grand Rapids, MI, USA, 1993.

[11] M. M. Dacorogna, U. A. Muller, R. B. Olsen, and O. V. Pictet: Modelling Short-Term Volatility with GARCH and $\mathrm{HARCH}$ Models.

[12] M. Xu, P. Shang, and A. Lin, "Cross-correlation analysis of stock markets using EMD and EEMD," Physica A: Statistical Mechanics and Its Applications, vol. 442, pp. 82-90, 2016.

[13] A. Ferreira, M. Moore, and S. Mukherjee, "Expectation errors in the foreign exchange market," Journal of International Money and Finance, vol. 95, pp. 44-51, 2019.

[14] S. Khuntia and J. K. Pattanayak, "Evolving efficiency of exchange rate movement: an evidence from Indian foreign exchange market," Global Business Review, vol. 21, no. 4, pp. 956-969, 2020.

[15] X. Meng and C.-H. Huang, "The time-frequency co-movement of Asian effective exchange rates: a wavelet approach with daily data," The North American Journal of Economics and Finance, vol. 48, pp. 131-148, 2019.

[16] S. Qureshi and M. Aftab, "Exchange rate interdependence in ASEAN markets: a wavelet analysis," Global Business Review, p. $0972150920919371,2020$.

[17] E. Asafo-Adjei, D. Agyapong, S. K. Agyei, S. Frimpong, R. Djimatey, and A. M. Adam, "Economic policy uncertainty and stock returns of Africa: a wavelet coherence analysis," Discrete Dynamics in Nature and Society, vol. 2020, Article ID 8846507, 8 pages, 2020.
[18] M. C. Mariani, P. K. Asante, M. A. M. Bhuiyan, M. P. BeccarVarela, S. Jaroszewicz, and O. K. Tweneboah, "Long-range correlations and characterization of financial and volcanic time series," Mathematics, vol. 8, no. 3, p. 441, 2020.

[19] P. O. Junior, G. Tweneboah, and A. M. Adam, "Interdependence of major exchange rates in Ghana: a wavelet coherence analysis," Journal of African Business, vol. 20, no. 3, pp. 407-430, 2019.

[20] N. E. Huang, Z. Shen, S. R. Long et al., "The empirical mode decomposition and the Hilbert spectrum for nonlinear and non-stationary time series analysis," Proceedings of the Royal Society of London. Series A: Mathematical, Physical and Engineering Sciences, vol. 454, no. 1971, pp. 903-995, 1998.

[21] C. Liu, X. Sun, J. Wang, J. Li, and J. Chen, "Multiscale information transmission between commodity markets: an EMD-Based transfer entropy network," Research in International Business and Finance, vol. 55, p. 101318, 2021.

[22] Z. Wu and N. E. Huang, "Ensemble empirical mode decomposition: a noise-assisted data analysis method," Advances in Adaptive Data Analysis, vol. 1, no. 1, pp. 1-41, 2009.

[23] T. Schreiber, "Measuring information transfer," Physical Review Letters, vol. 85, no. 2, pp. 461-464, 2000.

[24] A. Montalto, L. Faes, and D. Marinazzo, "MuTE: a MATLAB toolbox to compare established and novel estimators of the multivariate transfer entropy," PLoS One, vol. 9, no. 10, p. e109462, 2014.

[25] X. Mao and P. Shang, "Transfer entropy between multivariate time series," Communications in Nonlinear Science and $\mathrm{Nu}$ merical Simulation, vol. 47, pp. 338-347, 2017.

[26] A. Renyi, Probability Theory, North-Holland Publishing Company, Amsterdam, Netherlands, 1970.

[27] Q. Ji, E. Bouri, D. Roubaud, and L. Kristoufek, "Information interdependence among energy, cryptocurrency and major commodity markets," Energy Economics, vol. 81, pp. 1042$1055,2019$.

[28] J.-B. Geng, Q. Ji, and Y. Fan, "The relationship between regional natural gas markets and crude oil markets from a multi-scale nonlinear granger causality perspective," Energy Economics, vol. 67, pp. 98-110, 2017.

[29] X. Sun, X. Chen, J. Wang, and J. Li, "Multi-scale interactions between economic policy uncertainty and oil prices in timefrequency domains," The North American Journal of Economics and Finance, vol. 51, p. 100854, 2020.

[30] P. Flandrin, G. Rilling, and P. Goncalves, "Empirical mode decomposition as a filter bank," IEEE Signal Processing Letters, vol. 11, no. 2, pp. 112-114, 2004.

[31] N.-S. Kim, K. Chung, S. Ahn, J. W. Yu, and K. Choi, "Denoising traffic collision data using ensemble empirical mode decomposition (EEMD) and its application for constructing continuous risk profile (CRP)," Accident Analysis \& Prevention, vol. 71, pp. 29-37, 2014.

[32] H. Li, T. Liu, X. Wu, and Q. Chen, "Application of EEMD and improved frequency band entropy in bearing fault feature extraction," ISA Transactions, vol. 88, pp. 170-185, 2019.

[33] R. Chen and B. Pan, "Chinese stock index futures price fluctuation analysis and prediction based on complementary ensemble empirical mode decomposition," Mathematical Problems in Engineering, vol. 2016, Article ID 3791504, 13 pages, 2016.

[34] S. Behrendt, T. Dimpfl, F. J. Peter, and D. J. Zimmermann, "RTransferEntropy-quantifying information flow between different time series using effective transfer entropy," Software, vol. 10, p. 100265, 2019. 
[35] A. M. Adam, "Susceptibility of stock market returns to international economic policy: evidence from effective transfer entropy of Africa with the implication for open innovation," Journal of Open Innovation: Technology, Market, and Complexity, vol. 6, no. 3, p. 71, 2020.

[36] R. V. L. Hartley, "Transmission of Information," Bell System technical journal, vol. 7, no. 3, pp. 535-563, 1928.

[37] C. E. Shannon, "A mathematical theory of communication," Bell System Technical Journal, vol. 27, no. 3, pp. 379-423, 1948.

[38] S. Kullback and R. A. Leibler, "On information and sufficiency," The Annals of Mathematical Statistics, vol. 22, no. 1, pp. 79-86, 1951.

[39] C. Beck and F. Schögl, Thermodynamics of Chaotic Systems: An Introduction (No. 4), Cambridge University Press, Cambridge, UK, 1995.

[40] R. Marschinski and H. Kantz, "Analysing the information flow between financial time series," The European Physical Journal B, vol. 30, no. 2, pp. 275-281, 2002.

[41] T. Dimpfl and F. J. Peter, "The impact of the financial crisis on transatlantic information flows: an intraday analysis," Journal of International Financial Markets, Institutions and Money, vol. 31, pp. 1-13, 2014.

[42] J. S. Corrales, P. A. Imam, S. Weber, and E. Yehoue, "Dollarisation in sub-saharan Africa," Journal of African Economies, vol. 25, no. 1, pp. 28-54, 2016.

[43] P. J. J. Luukko, J. Helske, and E. Räsänen, "Introducing libeemd: a program package for performing the ensemble empirical mode decomposition," Computational Statistics, vol. 31, no. 2, pp. 545-557, 2016.

[44] E. Asafo-Adjei, A. M. Adam, and P. Owusu Junior, "Information flow between global equities and cryptocurrencies: a VMD-based entropy evaluating shocks from COVID-19 pandemic," Complexity, vol. 2021, Article ID 753753, 25 pages, 2021.

[45] S. Zhou, "The forward premium anomaly and the trend behavior of the exchange rates," Economics Letters, vol. 76, no. 2, pp. 273-279, 2002.

[46] M. Doyle, Internationalism: Kant, Ways of War and Peace, New York, NY, USA, 1997.

[47] S. W. Polachek, "Conflict and trade," Journal of Conflict Resolution, vol. 24, no. 1, pp. 55-78, 1980.

[48] T. M. Mokoena, R. Gupta, and R. Van Eyden, "Testing for PPP using SADC real exchange rates," South African Journal of Economics, vol. 77, no. 3, pp. 351-362, 2009.

[49] T. Qabhobho, C. Wait, and P. Le Roux, "Exchange rate volatility, the contagion and spillover effect from South African to other SADC currency markets: 2007-2015," African Journal of Business and Economic Research, vol. 15, no. 2, pp. 7-23, 2020.

[50] E. Anoruo and Y. Ahmad, "Regime-dependent monetary policy convergence: the case of southern African development community (SADC)," The Journal of Developing Areas, vol. 47, pp. 75-90, 2013.

[51] N. Duma, The Determinants of Exchange Rate Flexibility and the Theory of Optimum Currency Areas: An Application to SADC (Southern African Development Community), Doctoral dissertation, University of Natal, Durban, South Africa, 2001.

[52] E. H. Redda and P. F. Muzindusti, "Does SADC constitute an optimum currency area? Evidence from generalised purchasing power parity," in Proceedings of the Economics and Finance Conferences, Charleston, SC, USA, July 2017. 